\title{
A linear stability analysis of large-Prandtl-number thermocapillary liquid bridges
}

\author{
B. Xun ${ }^{a, c, *}$ P. G. Chen ${ }^{b}$ K. Li ${ }^{a}$ Z. Yin ${ }^{a}$ W. R. Hu ${ }^{a}$ \\ ${ }^{a}$ National Microgravity Laboratory, Institute of Mechanics, Chinese Academy of \\ Sciences, Beijing 100080, China \\ ${ }^{\mathrm{b}}$ Laboratoire Modélisation et Simulation Numérique en Mécanique et Génie des \\ Procédés, UMR 6181, CNRS-Universités d'Aix-Marseille, F-13451 Marseille \\ Cedex 20, France \\ c The Graduate School of Chinese Academy of Sciences, Beijing 100080, China
}

\begin{abstract}
A linear stability analysis is applied to determine the onset of oscillatory thermocapillary convection in cylindrical liquid bridges of large Prandtl numbers $(4 \leqslant$ $P r \leqslant 50)$. We focus on the relationships between the critical Reynolds number $R e_{c}$, the azimuthal wave number $m$, the aspect ratio $\Gamma$ and the Prandtl number $P r$. A detailed $R e_{c}-P r$ stability diagram is given for liquid bridges with various $\Gamma$. In the region of $\operatorname{Pr}>1$, which has been less studied previously and where $R e_{c}$ has been usually believed to decrease with the increase of $P r$, we found $R e_{c}$ exhibits an early increase for liquid bridges with $\Gamma$ around one. From the computed surface temperature gradient, it is concluded that the boundary layers developed at both solid ends of liquid bridges strengthen the stability of basic axisymmetric thermocapillary convection at large Prandtl number, and that the stability property of the basic flow is determined by the "effective" part of liquid bridge.
\end{abstract}

Key words: liquid bridge, instability, thermocapillary convection, critical Reynolds number, boundary layer PACS: 47.20.Dr, 47.27.Te, 47.15.Fe

* Corresponding author.

Email addresses: xunbo@imech.ac.cn (B. Xun), chen@L3M.univ-mrs.fr (P. G. Chen), likai@imech.ac.cn (K. Li), zhaohua.yin@imech.ac.cn (Z. Yin), wrhu@imech.ac.cn (W. R. Hu). 


\section{INTRODUCTION}

The instability of thermocapillary convection in liquid bridges has been studied extensively in the last decade due to its fundamental interest in transition process and its importance in floating-zone material processing, in particular under microgravity conditions. For simplification, early theoretical investigation on thermocapillary instability was focused on infinite liquid bridges (Xu and Davis $(1983,1984))$. However, the critical Marangoni numbers obtained from this model are much smaller than those obtained in laboratory experiments. Theoretical analyses of thermocapillary convection and small surface deformations in finite liquid bridges were provided by Kuhlmann (1989) and Chen and Roux (1991).

A number of experimental works on thermocapillary flow in liquid bridges have been carried out by several research groups (Chun and Wuest, 1978; Schwabe et al., 1978; Chun and Wuest, 1979; Chun, 1980; Velten et al., 1991; Kamotani et al., 1984; Yao et al., 1996; Preisser et al., 1983). The experimental evidence of thermocapillary flow in a liquid bridge was given by Chun and Wuest (1978) and by Schwabe et al. (1978). Chun and Wuest (1979) studied the flow transition from steady state to oscillatory state in a liquid bridge under reduced gravity condition. Chun (1980) found an S-shape distribution of the temperature on free surface before the onset of oscillation. A microgravity experiment of liquid bridge with varying aspect ratios during the D2-Spacelab mission was reported by Carotenuto et al. (1998). Yao et al. (1997) studied the oscillatory features using the drop shaft facility of Japan Microgravity Center. Very recently, Schwabe (2005) studied the instability of thermocapillary flow in a liquid bridge with aspect ratio near the Rayleigh limit (aspect ratio $\Gamma=5)$ during the flight of the sounding rocket MAXUS-4. By measuring the temperature distribution on free surface with thermocouple, Schwabe found that the critical Marangoni number would be much closer to theoretical result if the temperature gradient at the middle point of free surface instead of the mean gradient of whole free surface is used.

Meanwhile, there are also many numerical works devoted to the thermocapillary flow and its instability in liquid bridges during the last decade, e.g. direct three-dimensional numerical simulations (Levenstam and Amberg, 1995; Leypoldt et al., 2000; Savino and Monti, 1996), energy stability analyses (Shen et al., 1990; Neitzel et al., 1991), and linear stability analyses (Neitzel et al., 1993; Kuhlmann and Rath, 1993; Wanschura et al., 1995; Chen et al., 1997; Levenstam et al., 2001).

It is now well established that a steady, axisymmetric (2D) thermocapillary convection loses its stability first to a steady asymmetric (3D) flow and then to an oscillatory flow in liquid bridges of small Prandtl numbers $(\operatorname{Pr} \lesssim 0.06)$, 
while it loses its stability directly to an oscillatory flow (3D) in liquid bridges of large Prandtl numbers (up to $\mathrm{Pr}=7$ ) (Levenstam et al., 2001). The mechanism responsible for instability is found to be either purely hydrodynamic in nature or hydrothermal wave type, depending on the Prandtl number of the liquid (Wanschura et al., 1995; Chen et al., 1997). In the range of intermediate Prandtl numbers $(0.05 \lesssim P r \lesssim 0.85)$, the two mechanisms counteract with each other resulting in a drastic increase of critical Reynolds number $R e_{c}$ (Levenstam et al., 2001).

To our knowledge, the stability property when $\operatorname{Pr}>10$ has not yet been studied in detail, presumably for two reasons. First, it's more difficult to compute the thermocapillary flow in liquid bridges of large Prandtl numbers because a relatively high numerical resolution is required to resolve thin boundary layers. Second, it has been believed that the stability boundaries in the range of large Prandtl numbers would keep decreasing monotonously according to the results for $1.0 \lesssim \operatorname{Pr} \lesssim 7.0$, as shown by Levenstam et al. (2001).

In this paper we show that the stability boundaries exhibit different behaviors in different ranges of large Prandtl numbers. More specifically, an unexpected increase of $R e_{c}$ related to the boundary layers formed at both solid ends is found. Sections 2 contains a brief description of governing equations and numerical methods. Section 3 presents numerical results and offers a discussion on behaviors of $R e_{c}$ and $M a_{c}$ for liquid bridges of different $\operatorname{Pr}$. Finally, conclusions are given in Section 4.

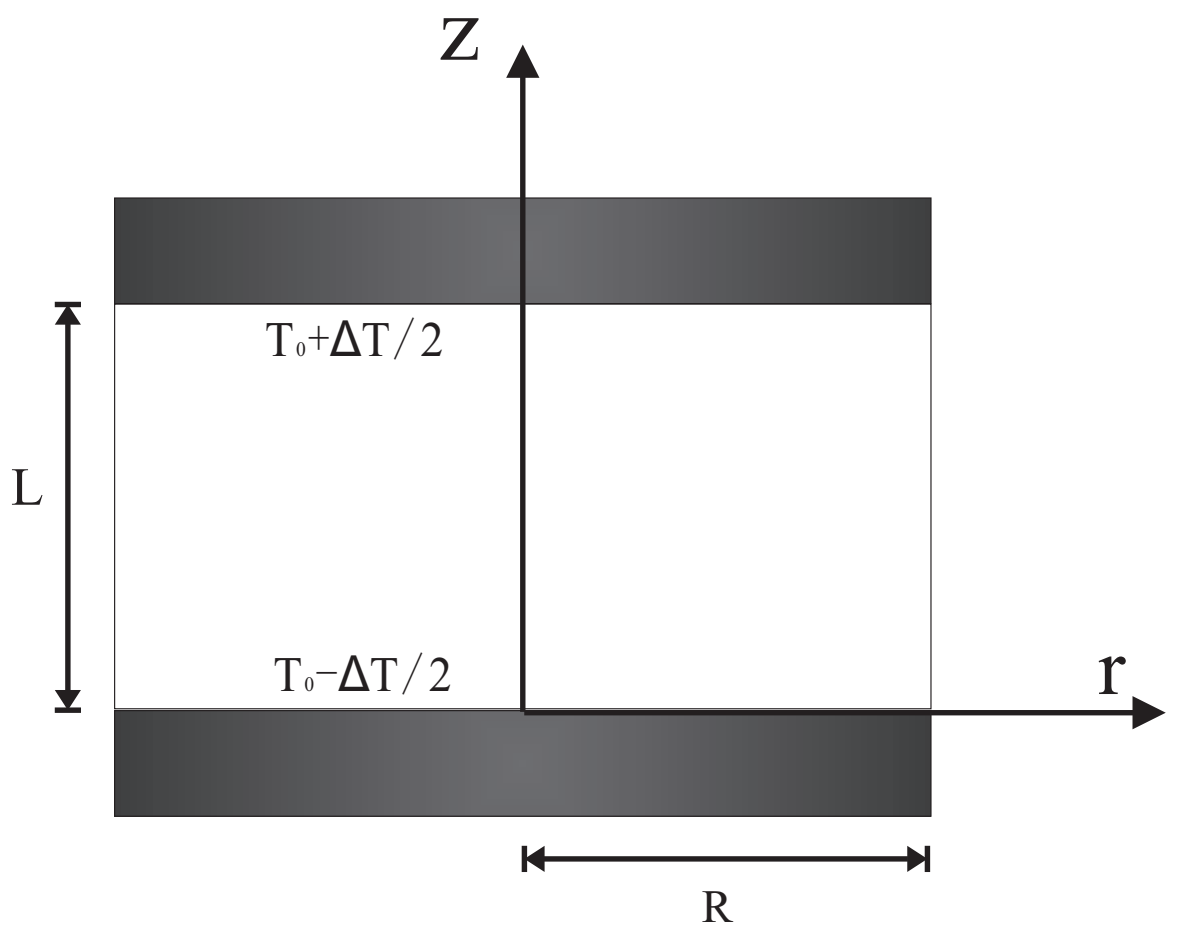

Fig. 1. Schematic of a floating half zone bridge. 


\section{Governing Equations and Solution Methods}

We consider a cylindrical half-zone liquid bridge of height $L$ and radius $R$. An imposed temperature difference $\Delta T$ is applied between two solid ends (Fig. 1). The length, velocity, pressure and time are scaled by $R, \frac{\gamma \Delta T}{\mu}, \frac{\gamma \Delta T}{R}$ and $\frac{R^{2}}{\nu}$ respectively, and the temperature measured with respect to $T_{0}$ is scaled by $\Delta T$, where $T_{0}$ is the mean temperature of the upper and lower ends, $\mu$ the dynamic viscosity coefficient, $\nu$ the kinematic viscosity coefficient, and $\gamma$ the negative temperature gradient of surface tension. In the cylindrical coordinate $(r, \theta, z)$, the thermocapillary flow in a cylindrical liquid bridge is governed, in dimensionless form, by the following equations (under microgravity condition):

$$
\begin{aligned}
& \nabla \cdot \mathbf{v}=0 \\
& \frac{\partial \mathbf{v}}{\partial t}+\operatorname{Re}(\mathbf{v} \cdot \nabla) \mathbf{v}=-\nabla p+\nabla^{2} \mathbf{v} \\
& \frac{\partial T}{\partial t}+\operatorname{Re}(\mathbf{v} \cdot \nabla) T=\frac{1}{P r} \nabla^{2} T
\end{aligned}
$$

where $\mathbf{v}=(u, v, w)$ denotes the velocity vector in the cylindrical coordinate, $p$ the pressure, $T$ the temperature, $R e=\frac{\gamma \Delta T R}{\mu \nu}$ the Reynolds number and $\operatorname{Pr}=\nu / \alpha$ the Prandtl number.

The boundary conditions are as follows:

$$
\begin{aligned}
& \mathbf{v}=0, T=\frac{1}{2}, \text { on } z=\Gamma, \\
& \mathbf{v}=0, T=-\frac{1}{2}, \text { on } z=0, \\
& u=0, \frac{\partial T}{\partial r}=0, \text { on } r=1, \\
& \frac{\partial w}{\partial r}+\frac{\partial T}{\partial z}=0, \frac{\partial v}{\partial r}-\frac{v}{r}+\frac{1}{r} \frac{\partial T}{\partial \theta}=0, \text { on } r=1,
\end{aligned}
$$

where $\Gamma=L / R$ is the aspect ratio of the liquid bridge.

As in Wanschura et al. (1995), the free surface condition Eq. (7) is changed into:

$$
\frac{\partial w}{\partial r}+\frac{\partial T}{\partial z} f(z)=0, \frac{\partial v}{\partial r}-\frac{v}{r}+\frac{1}{r} \frac{\partial T}{\partial \theta} f(z)=0, \text { on } r=1
$$


where

$$
f(z)=\left\{\begin{array}{cc}
\frac{1}{4}[1-\cos (10 \pi z)]^{2} & \text { for } 0 \leqslant z \leqslant 0.1 \\
1 & \text { for } 0.1 \leqslant z \leqslant 0.9 \\
\frac{1}{4}[1-\cos (10 \pi(1-z))]^{2} & \text { for } 0.9 \leqslant z \leqslant 1.0
\end{array}\right.
$$

In this work, both cases were considered, namely the original condition Eq. (7) and the modified condition Eq. (8).

The basic steady axisymmetric state, denoted by

$$
\mathbf{X}=\left\{\mathbf{V}(r, z)=U \mathbf{e}_{r}+W \mathbf{e}_{z}, P(r, z), T(r, z)\right\}
$$

is first determined for a given set of parameters $(\operatorname{Re}, \operatorname{Pr}$ and $\Gamma)$, and then small three-dimensional disturbances are added to the basic state and linearized by neglecting high orders of disturbances (Kuhlmann and Rath, 1993; Chen et al., 1997; Neitzel et al., 1993). The disturbances are assumed to be in the normal mode:

$$
\left[\begin{array}{l}
\mathbf{v}^{\prime}(r, \theta, z, t) \\
p^{\prime}(r, \theta, z, t) \\
T^{\prime}(r, \theta, z, t)
\end{array}\right]=\left[\begin{array}{c}
\mathbf{v}^{\prime}(r, z) \\
p^{\prime}(r, z) \\
T^{\prime}(r, z)
\end{array}\right] \exp \left(\sigma^{(m)} t+i m \theta\right),
$$

where the variables with prime denote the disturbances, $m$ the azimuthal wave number, $\sigma^{(m)}$ the complex growth rate of the corresponding perturbation mode, and $i=\sqrt{-1}$.

The discrete form of the linearized equations can be written as a generalized eigenvalue problem

$$
\mathfrak{g}(\mathbf{x}, \mathbf{X}, R e, m, \operatorname{Pr}, \Gamma) \equiv \mathbf{A} \mathbf{x}=\sigma \mathbf{B} \mathbf{x},
$$

where $\mathbf{x} \equiv(u, i v, w, p, T)^{\mathbf{T}}$ denotes a vector consisting of disturbance velocity, pressure and temperature. $\mathbf{A}$ is a real-valued nonsymmetric matrix, while $\mathbf{B}$ is a (singular) real-valued diagonal matrix. The eigenvalues and related eigenfunctions of problem Eq. (11) are solved by the Arnoldi method (Golub and Van Loan, 1996). The critical Reynolds number $R e_{c}$ is obtained for a given $\operatorname{Pr}$ when the maximal real part of $\sigma^{(m)}$ for all $m$ is zero.

It is worth noting that if we use the governing equations in the form of Eqs. (1-3), then the matrix $\mathbf{B}=\operatorname{diag}\{\cdots-1-1-1-1 \quad 0 \cdots\}$ will only have one 
class of nonzero values, namely -1 coming from Eqs. $(2,3)$; zero values in the diagonal being related to Eq. (1) and to the boundary conditions Eqs. (4-7). However, if we use another form of the energy equation Eq. (3) in our calculation, i.e.:

$$
\operatorname{Pr}\left[\frac{\partial T}{\partial t}+\operatorname{Re}(\mathbf{v} \cdot \nabla) T\right]=\nabla^{2} T
$$

then we will obtain a diagonal matrix $\mathbf{B}=\operatorname{diag}\{\cdots-1-1-1-\operatorname{Pr} 0 \cdots\}$ with two nonzero values $(-1$ and $-P r)$, coming from Eq. (2) and Eq. (12) respectively. The similar situation appears with matrix $\mathbf{A}$. Therefore, when $\operatorname{Pr} \gg 1$, the elements of the corresponding rows of $\mathbf{A}$ and $\mathbf{B}$ are much larger than the other rows; the numerical property of the matrixes would become very bad, making the computations very difficult. Theoretically speaking, the calculated eigenfunctions of problem Eq. (11) using Arnoldi method should be orthogonal. By examining the orthogonal property of the calculated eigenfunctions of the eigenvalue problem Eq. (11), it is found that our numerical code by using Eq. (3) provides much better results compared to that by using Eq. (12) for large $P r$.

In order to well resolve the boundary layers at both ends, a nonuniform grid with denser points near both solid ends and free surface is used in this work. Fig. 2 shows an example of the grid points distribution when the number of the grid points are $N_{r}=21$ and $N_{z}=36$ at the radial and axial direction respectively. The number of grid points we used in the calculation is, however, much larger: typically with $N_{r} \times N_{z}=91 \times 125$ for $\Gamma=1$.

The numerical scheme used in the present work is essentially the same as the previous one used by Chen et al. (1997), except that the energy Eq. (12) was replaced by Eq. (3) in order to obtain a better performance of numerical scheme for large $\mathrm{Pr}$. As a further verification of validation of our numerical code, we reproduced some calculations of thermocapillary convection in liquid bridge both for small and large Prandtl for which relatively accurate numerical results exist in the literature, and the results are in good agreement. For example, at $\operatorname{Pr}=0.1$ and $\Gamma=1$, we obtained $R e_{c}=16250$, close to $R e_{c}=$ 16094 given by Levenstam et al. (2001). 


\section{RESULTS AND DISCUSSIONS}

\subsection{Liquid bridge with $\Gamma=1$}

We report first the numerical results for a liquid bridge of unit aspect ratio. Fig. 3 shows the $R e_{c}-\operatorname{Pr}$ plot for $\operatorname{Pr} \geqslant 4$ according to the computed results listed in Tab. 1. As shown in Levenstam et al. (2001), $R e_{c}$ is very large at intermediate Prandtl numbers $(0.05 \lesssim \operatorname{Pr} \lesssim 0.85)$, and monotonously decreases with increasing Prandtl number when $\operatorname{Pr} \gtrsim 0.85$. Indeed, this behavior is also found in this work when $\operatorname{Pr}<8$. However, our numerical results reveal that for $\operatorname{Pr} \gtrsim 8, R e_{c}$ first increases with increasing Prandtl number and then decreases, which exhibits a local maximum around $\operatorname{Pr} \approx 28$. Correspondingly, the critical azimuthal wave number $m$ changes from $m=2$ to $m=1$ around $\operatorname{Pr} \approx 28$. Fig. 4 shows the critical Marangoni number $M a_{c}\left(=R e_{c} \operatorname{Pr}\right)$ as a function of Prandtl number. It is seen that $M a_{c}$ increases approximately linearly with increasing Prandtl number for the same $m$, but with different slopes for different $m$.

Fig. 5 shows the free surface temperature distribution of the basic steady axisymmetric state at the critical Reynolds number $R e_{c}$ for different $\operatorname{Pr}$. The S-shape temperature distributions, first reported by Chun (1980) in his experiment, can be clearly noted. The temperature gradient is steep in the boundary layers formed at the solid ends, and very small for the most part of the free sur-

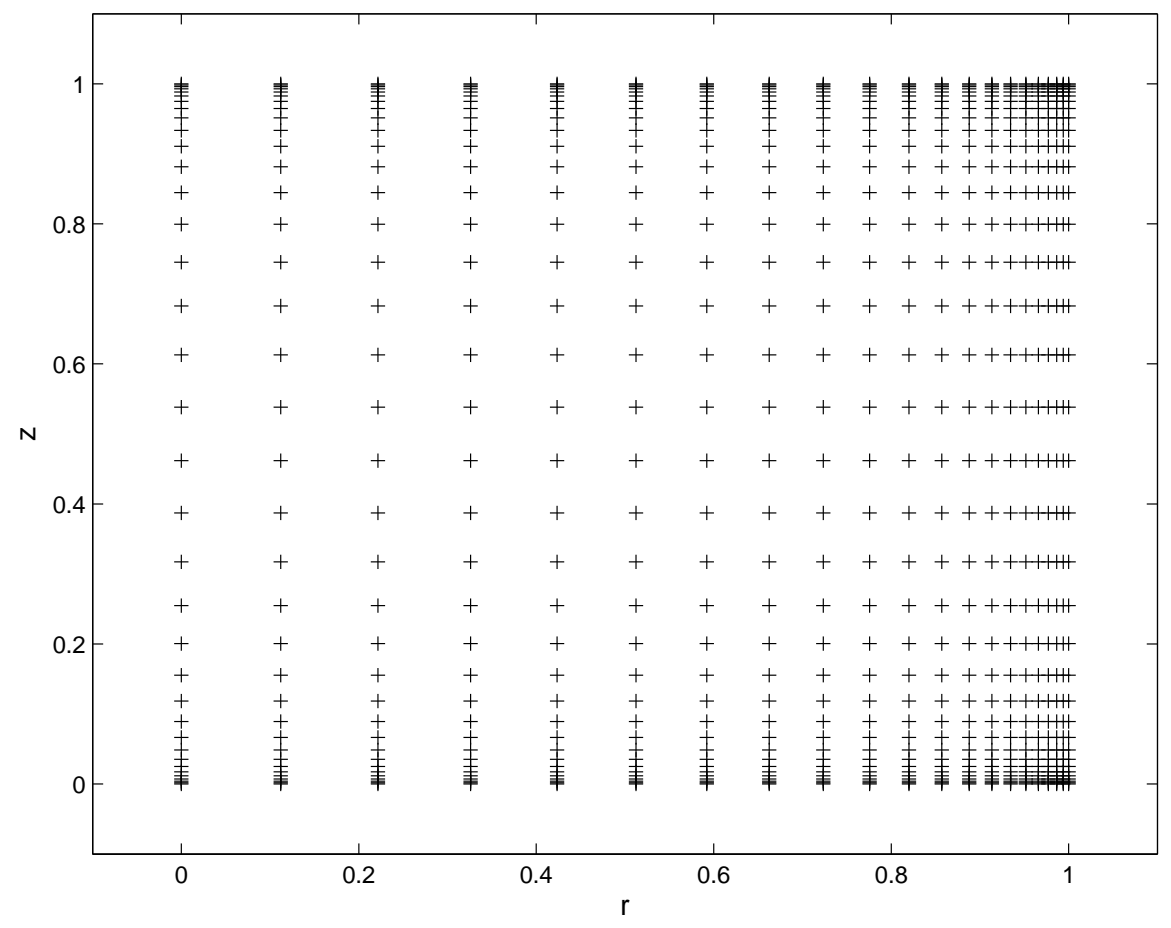

Fig. 2. An example of distribution of the grid points with $N_{r}=21$ and $N_{z}=36$. 


\begin{tabular}{|c|c|c||c|c|c|}
\hline $\operatorname{Pr}$ & $R e_{c}$ & $m$ & $P r$ & $R e_{c}$ & $m$ \\
\hline 4 & 1000 & 2 & 28 & 1310 & 2 \\
\hline 6 & 890 & 2 & 30 & 1300 & 1 \\
\hline 8 & 870 & 2 & 35 & 1175 & 1 \\
\hline 10 & 905 & 2 & 40 & 1075 & 1 \\
\hline 15 & 1095 & 2 & 45 & 1000 & 1 \\
\hline 20 & 1227 & 2 & 50 & 930 & 1 \\
\hline 25 & 1285 & 2 & & & \\
\hline
\end{tabular}

Table 1

Computed critical Reynolds number $R e_{c}$ and the corresponding azimuthal wave number $m$ as a function of Prandtl number $\operatorname{Pr}$ for $\Gamma=1$.

face (see also Fig. 6). For instance, the temperature difference between $z=0.1$ and $z=0.9$ is less than $\frac{\Delta T}{10}$ at $\operatorname{Pr}=15\left(R e_{c}=1095\right)$. The boundary layers become thinner with steeper temperature gradient inside it with increasing Prandtl number. Correspondingly, the temperature gradient at the rest of the free surface decreases. The axial velocity distributions on the free surface as shown in Fig. 7 exhibit similar behavior with increasing Prandtl number.

Since the disturbances must satisfy the no-slip boundary conditions at the

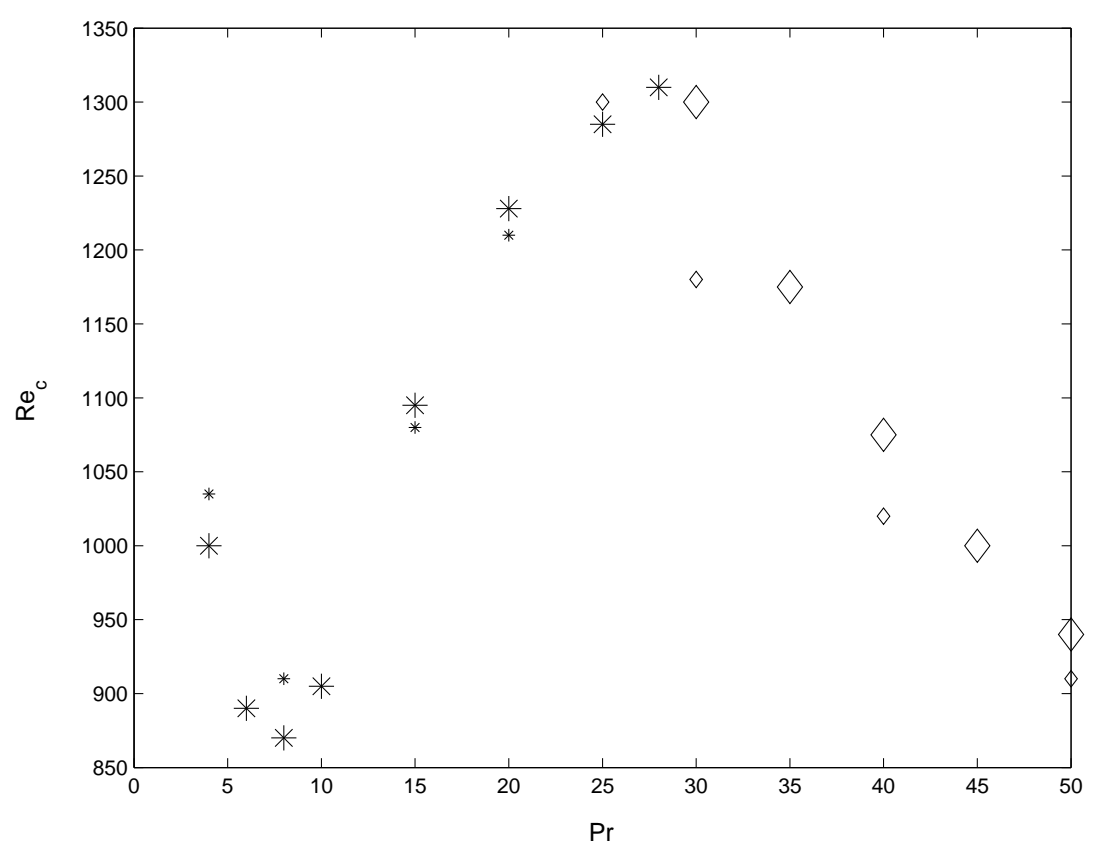

Fig. 3. $R e_{c}$ versus $\operatorname{Pr}$ for $\Gamma=1$. The points " $*$ " correspond to the critical azimuthal wave number $m=2$, and the diamond $m=1$. The points with double size correspond to the original free surface condition Eq. (7), others correspond to the modified condition Eq. (8). 


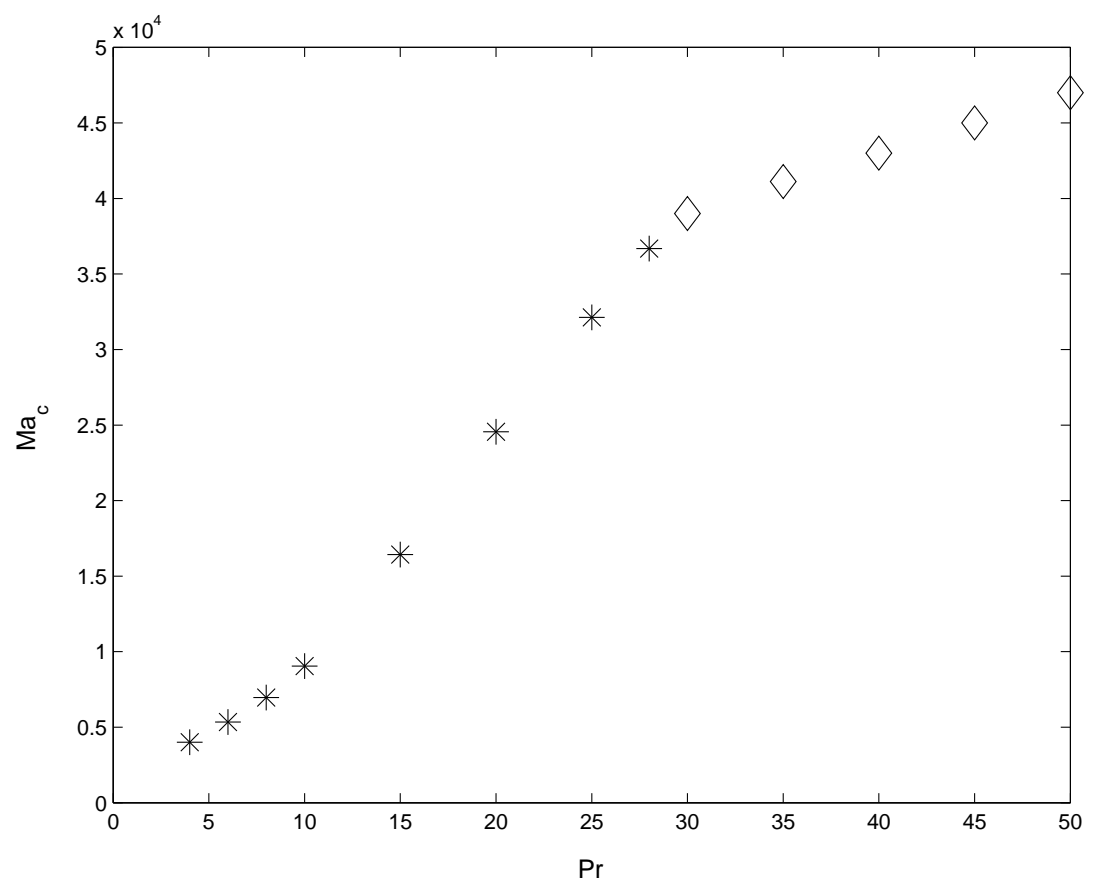

Fig. 4. $M a_{c}$ versus $\operatorname{Pr}$ for $\Gamma=1$. The points "*" correspond to the critical azimuthal wave number $m=2$, and the diamond $m=1$.

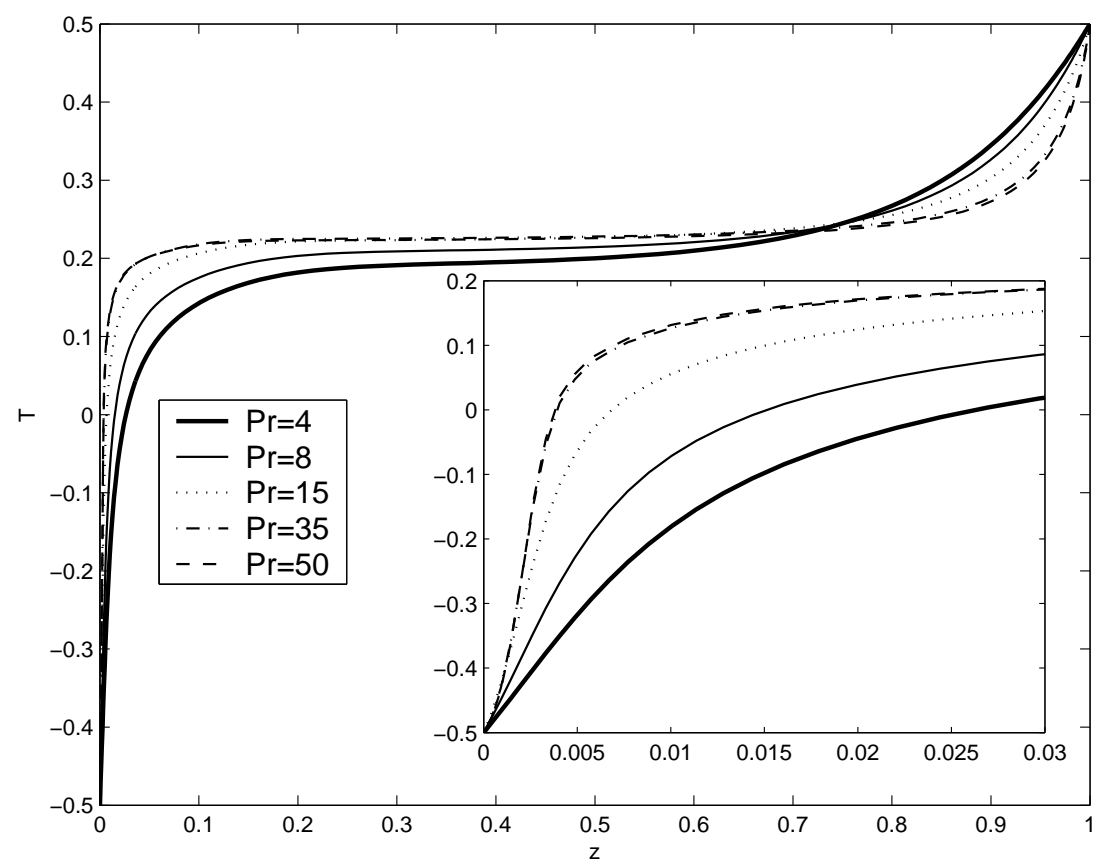

Fig. 5. Temperature distribution on free surface at the corresponding $R e_{c}$ for different $\mathrm{Pr}$.

solid ends, the absolute values of disturbances in the boundary layers should be smaller than those outside the boundary layers. This is indeed as illustrated in Fig. 8- 9 from the calculated eigenfunctions of the problem Eq. (11). It is 


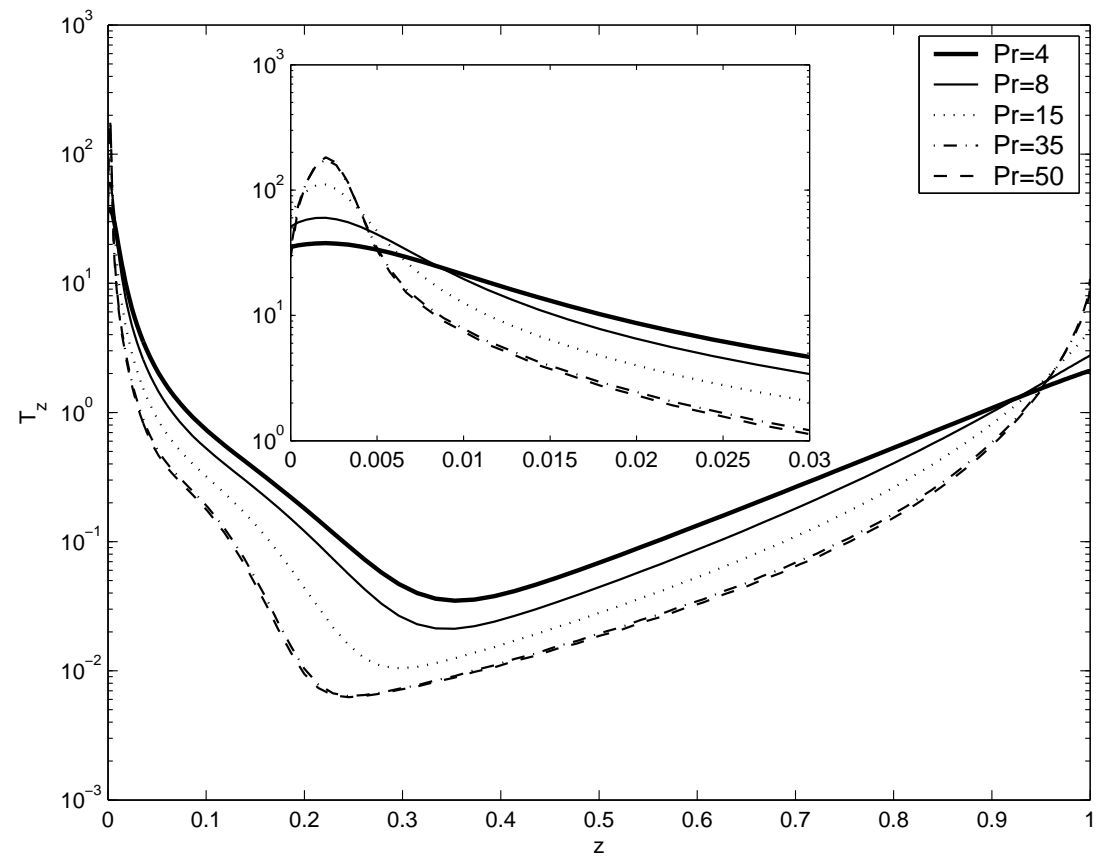

Fig. 6. Temperature gradient along the free surface at the corresponding $R e_{c}$ for different $\mathrm{Pr}$.

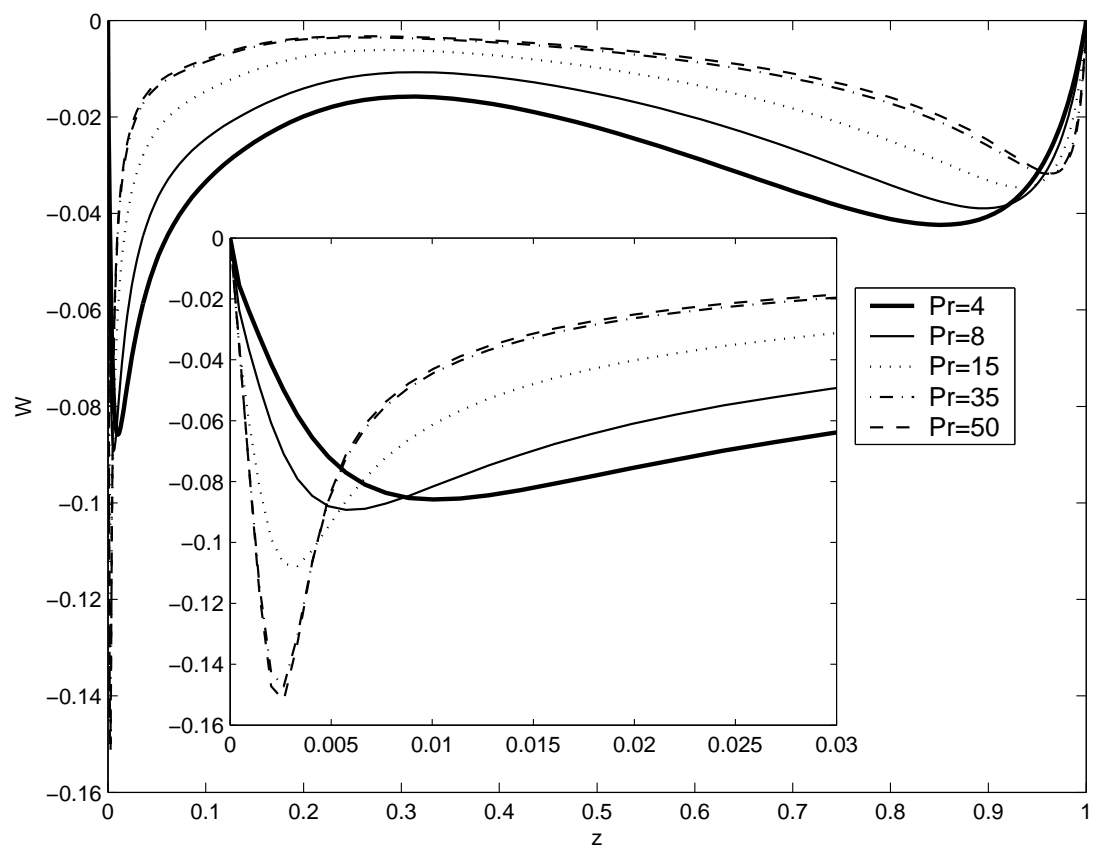

Fig. 7. Axial velocity $W$ on free surface at the corresponding $\operatorname{Re}_{c}$ for different $\operatorname{Pr}$.

noticed that the basic state loses its stability more easily with the disturbances of such eigenfunction since they correspond to the most unstable mode of disturbances. Therefore, the part of the liquid bridge with largest disturbances is the most unstable. As a good estimation, only the disturbances outside the thin boundary layers need to be taken into account (Schwabe, 2005). From 


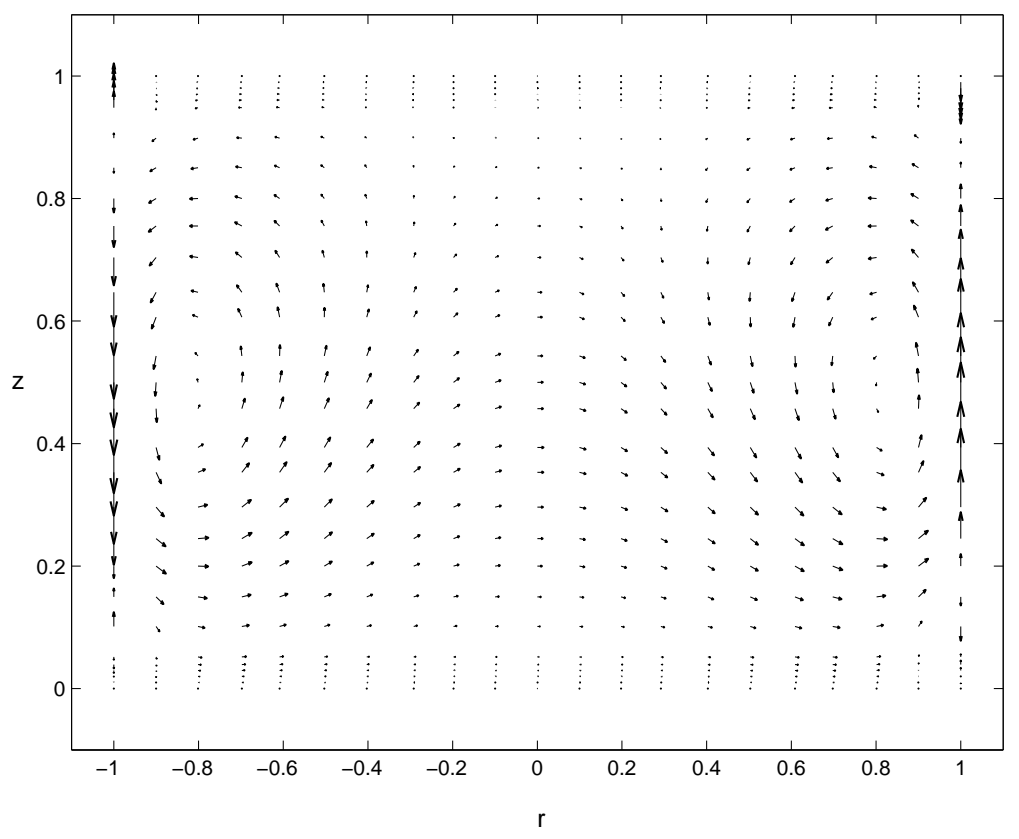

Fig. 8. Distribution of disturbance velocity on $\theta=0$ and $\pi$ of liquid bridge with $\Gamma=1.0$ at $\operatorname{Pr}=30$, where $m=1$.
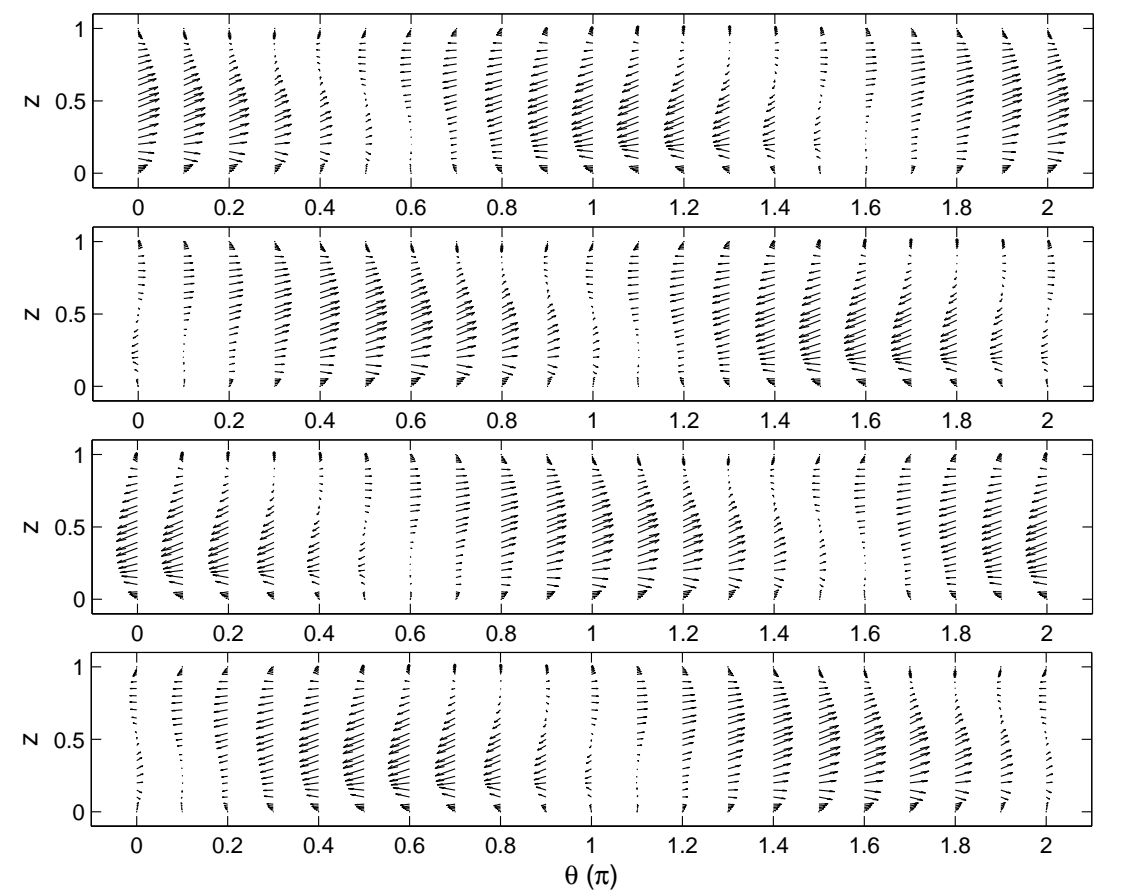

Fig. 9. Distribution of disturbance velocity on free surface of liquid bridge with $\Gamma=1.0$ at $\operatorname{Pr}=30$. where $m=1$. The time step between the pictures is a quarter of the oscillating period.

this point of view, it is useful to introduce the idea of an "effective" part of the liquid bridge in the analysis. More specifically, we use the temperature gradient at the middle part of free surface instead of $\frac{\Delta T}{L}$. In the following, 
the "effective" aspect ratio is denoted as $\Gamma_{e}$ and the "effective" temperature difference $(\Delta T)_{e}$.

As shown in Fig. 6, the dimensionless temperature difference $\frac{(\Delta T)_{e}}{\Delta T}$ decreases with increasing Prandtl number, and a larger $R e_{c}$ is needed to destabilize the thermocapillary flow. However, when $\operatorname{Pr}$ is larger than 22, the dimensionless temperature distribution remains nearly the same, and $R e_{c}$ drops down slowly with increasing Prandtl number. On the other hand, it is known that the critical azimuthal wave number $m$ is mostly determined by the aspect ratio (Preisser et al., 1983). The larger the aspect ratio (in our case, the effective aspect ratio), the smaller the critical azimuthal wave number. As shown in Fig. 5 and Fig. 7, the boundary layers at the two solid ends become thinner with larger $\operatorname{Pr}$, leading to a larger effective aspect ratio $\Gamma_{e}$, so that the critical azimuthal wave number is decreased from $m=2$ to $m=1$.

Finally, as shown in Fig. 3, it is interesting to note that the computed results obtained by using the original free surface condition and the modified free surface condition Eq. (8) do not differ much. A plausible explanation is that due to the elliptical property of the governing equations, the recension of the free surface condition near both solid ends does not affect the result at the middle part of the liquid bridge. As mentioned above, the transition from steady state to oscillatory state depends mainly on the middle part of the liquid bridge, thus the modified free surface condition does not affect the critical Reynolds number much.

\subsection{Liquid bridges of different aspect ratios}

We report here briefly the numerical results for liquid bridges of different aspect ratios. The computed results are shown in Tab. 2. Fig. 10 shows the $R e_{c^{-}}$ $\operatorname{Pr}$ plot for various $\Gamma$. The differences between the $R e_{c}-\operatorname{Pr}$ plot for various $\Gamma$ are the location of the local maximum region of $R e_{c}$ and the critical azimuthal wave number. For example, with $\Gamma=1.2, R e_{c}$ increases when $6 \lesssim \operatorname{Pr} \lesssim 15$. There is a local maximum around $\operatorname{Pr} \approx 15$, and the critical azimuthal wave number changes from $m=2$ to $m=1$ with the increase of $\operatorname{Pr}$ around $\operatorname{Pr} \approx 15$. The peak of $R e_{c}$ in this region is sharper than that with $\Gamma=1$.

The aspect ratio affects the critical Reynolds number and the critical azimuthal wave number. Larger the aspect ratio, smaller the $R e_{c}$ and $m$, with an early increase region of $R e_{c}$ and the location of the local maximum. It is found that the early increase of $R e_{c}$ is sharper for larger aspect ratio, which may be associated with the quicker development of the boundary layers in smaller increase region of $\operatorname{Pr}$. A further study is needed to explain this behavior. 


\begin{tabular}{|c|c|c|c||c|c|c|c|}
\hline $\operatorname{Pr}$ & $\Gamma$ & $R e_{c}$ & $m$ & $\operatorname{Pr}$ & $\Gamma$ & $R e_{c}$ & $m$ \\
\hline 4 & 1.2 & 940 & 2 & 50 & 1.2 & 840 & 1 \\
\hline 6 & 1.2 & 895 & 2 & 4 & 0.6 & 1885 & 4 \\
\hline 8 & 1.2 & 965 & 2 & 8 & 0.6 & 1398 & 3 \\
\hline 10 & 1.2 & 1133 & 2 & 15 & 0.6 & 1280 & 3 \\
\hline 15 & 1.2 & 1540 & 1 & 20 & 0.6 & 1310 & 3 \\
\hline 20 & 1.2 & 1285 & 1 & 25 & 0.6 & 1350 & 3 \\
\hline 25 & 1.2 & 1140 & 1 & 30 & 0.6 & 1385 & 3 \\
\hline 30 & 1.2 & 1040 & 1 & 35 & 0.6 & 1415 & 3 \\
\hline 35 & 1.2 & 970 & 1 & 40 & 0.6 & 1440 & 3 \\
\hline 40 & 1.2 & 917 & 1 & 45 & 0.6 & 1390 & 2 \\
\hline 45 & 1.2 & 875 & 1 & 50 & 0.6 & 1338 & 2 \\
\hline
\end{tabular}

Table 2

Computed critical Reynolds number $R e_{c}$ and the corresponding azimuthal wave number $m$ as a function of Prandtl number $\operatorname{Pr}$ for different aspect ratios $\Gamma$.

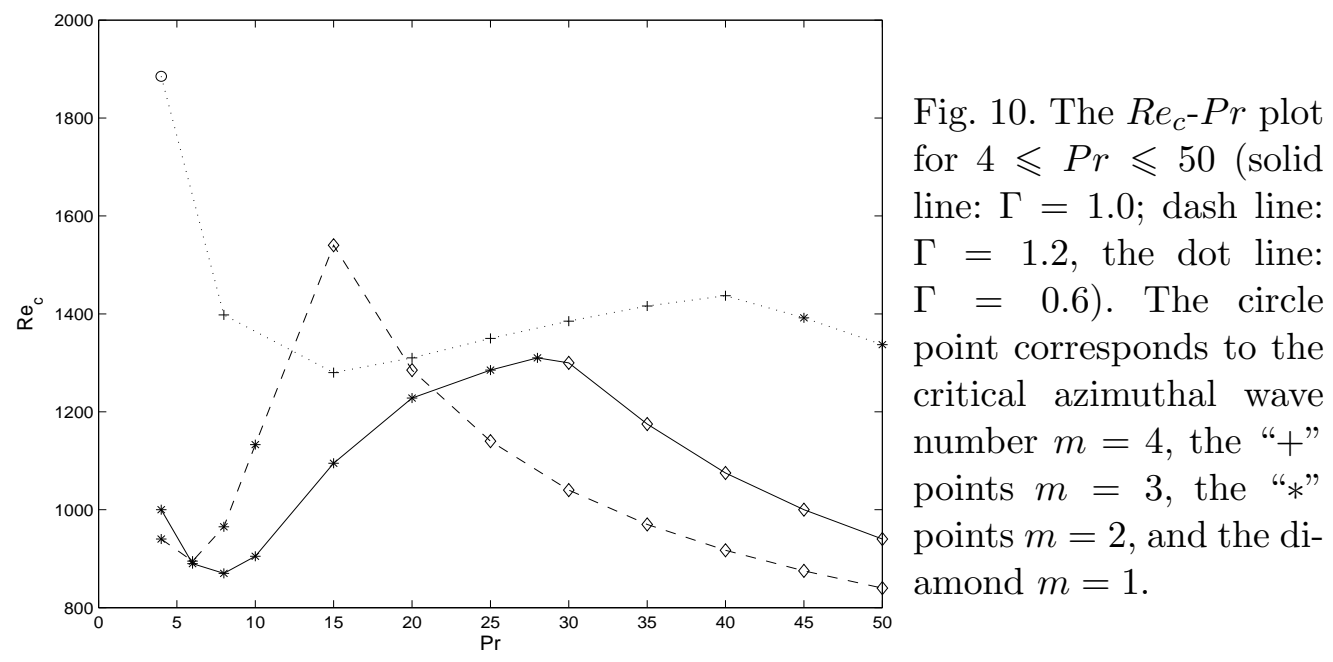

\section{CONCLUSIONS}

In conclusion, extended plots of $R e_{c}$ and $M a_{c}$ versus $\operatorname{Pr}$ have been obtained for cylindrical liquid bridges of different aspect ratios. For liquid bridge with unit aspect ratio, we found an unexpected increase of $\operatorname{Re}_{c}$ around $8 \lesssim \operatorname{Pr} \lesssim 22$, which is associated with the development of boundary layers at both solid ends. The behavior of $R e_{c}$ and $m$ relies more on the "effective" part of liquid bridge beyond the boundary layers. The effect of the aspect ratio is also studied. The aspect ratio doesn't change the qualitative features of the $R e_{c}$ versus $\operatorname{Pr}$ plot, but only some quantitative features, such as the early increase region of $R e_{c}$. 
The present work has been focused on cylindrical liquid bridges, however, it is well known that the shape of the free surface, or the volume of the liquid bridge is another important parameter of the thermocapillary flow (Chen and $\mathrm{Hu}$, 1998; Chen et al., 1999; Nienhuser and Kuhlmann, 2002; Hu and Tang, 2003; Shevtsova, 2005). Therefore, the variation of the critical Reynolds number with Prandtl number for different volume and aspect ratios, in particular in the case of large Prandtl number, remains to be investigated.

\section{ACKNOWLEDGEMENTS}

The authors wish to thank Professor Z.M. Tang for her helpful comments and discussions. This work was partially carried out when P.G.C visited the National Microgravity Laboratory/CAS in July 2005. The support from the National Foundation Nature Science of China (10432060 and 10502054) is gratefully acknowledged.

\section{References}

Carotenuto, L., Castagnolo, D., Albanese, C. et al. Instability of thermocapillary convection in liquid bridges. Phys. Fluids 10, 555-565, 1998.

Chen, G., Lizée, A., Roux, B. Bifurcation analysis of thermocapillary convection in cylindrical liquid bridges. J. Crystal Growth 180, 638-647, 1997.

Chen, G., Roux, B. An analytical study of thermocapillary flow and surface deformations in floating zones. Microgravity Quarterly 1, 73-80, 1991.

Chen, Q. S., Hu, W. R. Influence of liquid bridge volume on instability of floating half zone convection. Int. J. Heat Mass Transfer 41, 825-837, 1998.

Chen, Q. S., Hu, W. R., Prasad, V. Effect of liquid bridge volume on the instability in small-Prandtl-number half zones. J. Crystal Growth 203, 261268, 1999.

Chun, C. H. Experiments on steady and oscillatory temperature distribution in a floating zone due to the marangoni convection. Acta Astronautica 7, 479-488, 1980.

Chun, C. H., Wuest, W. A micro-gravity simulation of the marangoni convection. Acta Astronautica 5, 681-686, 1978.

Chun, C. H., Wuest, W. Experiments on the transition from the steady to the oscillatory marangoni-convection of a floating zone under reduced gravity effect. Acta Astronautica 6, 1073-1082, 1979.

Golub, G. H., Van Loan C F Matrix Computations. Johns Hopkins University Press, Baltimore, 1996.

$\mathrm{Hu}$, W. R., Tang, Z. M. Influence of liquid bridge volume on the floating zone convection. Cryst. Res. Technol. 38, 627-634, 2003. 
Kamotani, Y., Ostrach, S., Vargas, M. Oscillatory thermocapillary convection in a simulated floating-zone configuration. J. Crystal Growth 66, 83-90, 1984.

Kuhlmann, H. Small amplitude thermocapillary flow and surface deformations in a liquid bridge. Phys. Fluids A1, 672-677, 1989.

Kuhlmann, H. C., Rath, H. J. Hydrodynamic instabilities in cylindrical thermocapillary liquid bridges. J. Fluid Mech. 247, 247-274, 1993.

Levenstam, M., Amberg, G. Hydrodynamic instabilities of thermocapillary flow in a half-zone. J. Fluid Mech. 297, 357-372, 1995.

Levenstam, M., Amberg, G., Winkler, C. Instability of thermocapillary convection in a half-zone at intermediate Prandtl numbers. Phys. Fluids 13, 807-816, 2001.

Leypoldt, J., Kuhlmann, H. C., Rath, H. J. Three dimensional numerical simulation of thermocapillary flows in cylindrical liquid bridges. J. Fluid Mech. 414, 285-314, 2000.

Neitzel, G. P., Chang, K. T., Jankowski, D. F. et al. Linear stability theory of thermocapillary convection in a model of the float-zone crystal-growth process. Phys. Fluids A5, 108-114, 1993.

Neitzel, G. P., Law, C. C., Jankowski, D. F. et al. Energy stability of thermocapillary convection in a model of the float-zone crystal-growth process. II: Nonaxisymetric disturbances. Phys. Fluids A3, 2841-2846, 1991.

Nienhuser, CH., Kuhlmann, H. C. Stability of thermocapillary flows in noncylindrical liquid bridges. J. Fluid Mech. 458, 35-73, 2002.

Preisser, F., Schwabe D., Scharmann, A. Steady and oscillatory thermocapillary convection in liquid columns with free cylindrical surface. J. Fluid Mech. 126, 545-567, 1983.

Savino, R., Monti, R. Oscillatory marangoni convection in cylindrical liquid bridges. Phys. Fluids 8, 2906-2922, 1996.

Schwabe, D. Hydrothermal waves in a liquid bridge with aspect ratio near the Rayleigh limit under microgravity. Phys. Fluids 17, 112104, 2005.

Schwabe, D., Scharmann, A., Preisser, F. et al. Experiments on surface tension driven flow in floating zone melting. J. Crystal Growth 43, 305-312, 1978.

Shen, Y., Neitzel, G. P., Jankowski, D. F. et al. Energy stability of thermocapillary convection in a model of the float-zone crystal-growth process. J. Fluid Mech. 217, 639-660, 1990.

Shevtsova, V. Thermal convection in liquid bridges with curved free surfaces: Benchmark of numerical solutions. J. Crystal Growth 280, 632-651, 2005.

Velten, R., Schwabe, D., Scharmann, A. The periodic instability of thermocapillary convection in cylindrical liquid bridges. Phys. Fluids A3, 267-279, 1991.

Wanschura, M., Shevtsova, V. M., Kuhlmann, H. C. et al. Convective instability mechanisms in thermocapillary liquid bridges. Phys. Fluids 7, 912-925, 1995.

Xu, J. J., Davis, S. H. Liquid bridges with thermocapillarity. Phys. Fluids 26, 2880-2886, 1983. 
$\mathrm{Xu}$, J. J., Davis, S. H. Convective thermocapillary instabilities in liquid bridges. Phys. Fluids 27, 1102-1107, 1984.

Yao, Y. L., Liu, F., Hu, W. R. How to determine critical marangoni number in half floating zone convection. Int. J. Heat Mass Transfer 39, 2539-2544, 1996.

Yao, Y. L., Shu, J. Z., Xie, J. C. et al. Transition of oscillatory floating half zone convection from Earth's gravity to microgravity. Int. J. Heat Mass Transfer 40, 2517-2524, 1997. 